\title{
A Study on Federated Cloud Computing Environment
}

\author{
Sapana Sanjay Bhuskute, Sujata Kadu
}

\begin{abstract}
Abstrac: Federated cloud computing is the advancement in the area of the general cloud computing paradigm. In a federated cloud environment, multiple cloud service providers share their computing assets, servers, and various facilities to fulfill customer demands. Federated cloud computing terminology consists of the aggregation of services considered by interoperability characteristics and creates the integration of several cloud service providers regardless of any geographical location. It improves the performance, utilization of facilities, minimizes response time and pricing model by partial subcontracting various computing resources and facilities from the nearby cost-efficient province. Customers also get profited from service level agreements signed between the cloud service providers through intermediator cloud brokers. This work aims to survey the federated cloud environment, its various architectural types, advantages associated with the federation, challenges associated with a federated cloud environment, and future research directions in the federated cloud computing research area.
\end{abstract}

Keywords: Cloud Architecture, Cloud Broker, Cloud Federation, Cloud Service Providers, Federated Cloud, Multicloud Deployment, SLA.

\section{INTRODUCTION}

Cloud Computing is a new technology that provides different types of computing resources and services as per the demands of the customer over the Web under a single service provider. Cloud computing follows a pay per demand service model that increases its popularity among customers. Cloud computing clouds are classified under three categories such as public, private, and hybrid clouds that provide various computing services in different formats of service models such as Platform as a Service (PaaS), Infrastructure as a Service (IaaS), and Software as a Service (SaaS). According to the Service Level Agreement (SLA), cloud computing services and resources are provided between the cloud service provider and customer [16]. In cloud computing various computing resources like servers, applications, networks, storage, and services can be quickly allotted and released with minimum interference with the cloud service provider [19].

Manuscript received on July 20, 2021.

Revised Manuscript received on July 27, 2021.

Manuscript published on 30 July, 2021.

* Correspondence Author

Sapana Sanjay Bhuskute*, Department of Information Technology, Terna Engineering College, Nerul, India, sapanabhuskute01@gmail.com

Prof. Sujata Kadu, Department of Information Technology, Terna Engineering College, Nerul, India, sujatakadu@ternaengg.ac.in

(c) The Authors. Published by Blue Eyes Intelligence Engineering and Sciences Publication (BEIESP). This is an open access article under the CC BY-NC-ND license (http://creativecommons.org/licenses/by-nc-nd/4.0/)
This methodology provides financial profits in terms of reduced adjustment of resource deployment through the aggregation of demands and reduced IT administration cost per customer because of multi-tenant structural design [4].

These large-scale profits provided by cloud computing lead to the industrial acceptance of cloud facilities. Industries nowadays prefer cloud services over traditional in-house IT coordination and facilities due to the more affordability and reliability provided by cloud services. But, along with benefits, cloud computing model also faces some downsides. Some customers don't want to outsource their business application and data into the cloud due to security reasons, lack of consistent service interfaces, vendor lock-in of protocols \& data formats, fear of losing ownership and control [1]. Along with all these single deployed clouds there is always fear of any natural catastrophe appearing suddenly or any security attacks bombarded on the network by hackers for their malicious motive can lead to data loss for customers [17]. All this concern causes inconvenient financial scenarios hence the need to find a more convenient solution which can overcome all these problems.

In this research work, the cloud federation concept is studied thoroughly to minimize vendor lock-in's adverse effects and design inter-operable \& flexible cloud-based software. Numerous inter-connected cloud models create a federated cloud that is handled and positioned by various interior and exterior cloud computing facilities to fulfill the requirements of the businesses [13]. Federated cloud contains many service providers laced at different geographical locations, who share their facilities \& servers to fulfill the customer's demand. Cloud federation work is based on the Infrastructure as a Service (IaaS) model, in which the customer demands the infrastructure and is fulfilled in the form of virtual machines loaded with different operating systems and software as per the demand of the customer [6].

In a federated cloud computing environment customers of one cloud facility can use the credential from one facility to use any other cloud facility without the need to sign in separately for that. Federated cloud also acts as a good choice in decentralized storage network conditions. Hence, many IT cloud service providers highly prefer the federated cloud computing concept due to ease in management and setting out of cloud computing facilities in diverse in-house and exterior clouds to fulfill the demands of various businesses [19].

The cloud service broker is the main key between the cloud service provider and a customer. This broker sends customer demands towards the cloud service provider and finds out the best cloud service provider to fulfill the demand of the customer $[2,18]$.

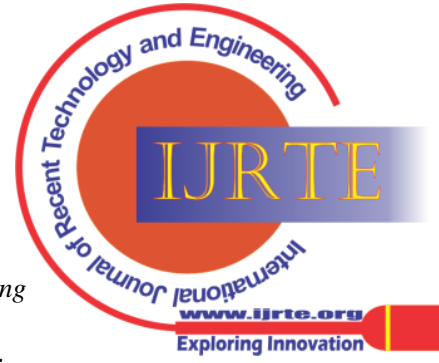


This broker does the work of management, monitoring, and assessment of the cloud service provider nonetheless of the geographical situation of the servers. When the best cloud service provider is decided by the broker who can fulfill the customer's demand, the Service Level Agreement (SLA) takes place to access all the required facilities [14]. This kind of engagement in a federated cloud environment provides a high level of Quality of Service (QoS) as compared to the single deployed cloud [20].

A federated cloud consists of various cloud service providers bound with regular SLAs. Every cloud service provider fulfills the demands of the customer depending upon the last resources. In a federated cloud environment, different types of clouds provide services to different applications. When private cloud resources are not able to fulfill the demand of the customer, then public cloud resources can be utilized to overcome this problem. The cloud service broker assigns resources from different cloud service providers placed at geographical locations to fulfill the customer demand [20].

\section{LITERATURE SURVEY}

In a federated cloud environment cloud service providers and customers both always try to maintain the optimal resource provisioning with minimum service cost for deployment of applications and without disturbing the Quality of Services (QoS). Table I represents the research performed by various authors on different types of federated cloud environments.

Table I. Literature Survey

\begin{tabular}{|c|c|c|}
\hline Author & Description & Conclusion \\
\hline $\begin{array}{l}\text { R. Van den } \\
\text { Bossche et al. } \\
\text { [3] }\end{array}$ & $\begin{array}{l}\text { It utilizes bursting } \\
\text { methodology } \\
\text { private to public } \\
\text { clouds to optimize } \\
\text { resource provisioning. }\end{array}$ & $\begin{array}{l}\text { Binary integer } \\
\text { model used in } \\
\text { cloud bursting } \\
\text { architecture of } \\
\text { federated cloud } \\
\text { environment for } \\
\text { cost optimization. }\end{array}$ \\
\hline $\begin{array}{l}\text { David } \\
\text { Breitgand } \\
\text { al. [5] }\end{array}$ & $\begin{array}{l}\text { It forms Aggregated } \\
\text { cloud architecture of a } \\
\text { federated cloud } \\
\text { environment to } \\
\text { provide assistance to } \\
\text { cloud service } \\
\text { providers for efficient } \\
\text { allocation of QoS. }\end{array}$ & $\begin{array}{l}\text { Load balancing } \\
\text { Greedy approach } \\
\text { and integer } \\
\text { programming } \\
\text { models used for } \\
\text { minimal } \\
\begin{array}{l}\text { consumption of } \\
\text { power. }\end{array}\end{array}$ \\
\hline $\begin{array}{l}\text { Christian } \\
\text { Vecchiola } \\
\text { al. [7] }\end{array}$ & $\begin{array}{l}\text { Aggregated cloud } \\
\text { architecture is used } \\
\text { for resources } \\
\text { provisioning from } \\
\text { shared cloud service } \\
\text { providers proficiently. } \\
\text { It also improves the } \\
\text { response time of } \\
\text { request management. }\end{array}$ & $\begin{array}{l}\text { The integer } \\
\text { programming } \\
\text { method used is for } \\
\text { performance } \\
\text { improvement in } \\
\text { the aggregated } \\
\text { federated cloud } \\
\text { environment. }\end{array}$ \\
\hline $\begin{array}{l}\text { Johan } \\
\text { Tordsson } \\
\text { al. [8] }\end{array}$ & $\begin{array}{l}\text { It uses two types of } \\
\text { methodologies for } \\
\text { resource provisioning } \\
\text { i.e. optimal placement } \\
\text { of virtual machines \& } \\
\text { monitoring, }\end{array}$ & $\begin{array}{l}\text { Binary integer and } \\
\text { tightly coupled } \\
\text { cloud broker } \\
\text { architecture } r \\
\text { federated cloud } \\
\text { used for cost }\end{array}$ \\
\hline
\end{tabular}

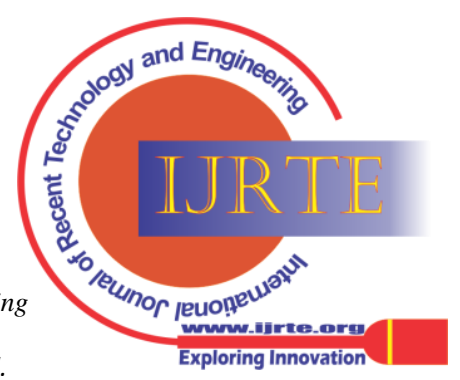

A federated cloud consists of services from numerous diverse cloud service providers combined at a single point. Federated cloud supports three basic features of interoperability such as asset migration, asset redundancy, and a combination of balancing assets. In that, asse contains repositioning of various computing dese data, program code, virtual machine drom one service provider to other service can use the same facility from different service providers concurrently or at the same time. The combination of balancing assets and facilities permits to combine the dissimilar sorts of combined facilities. 
The cloud federation concept provides various benefits to both the cloud service providers as well as to the customers of the cloud too. Customers mainly get benefits from the minimum price and good performance, while cloud service providers can offer improved cloud facilities to their all customers [4].

A federated cloud environment is made by various components such as customers, public cloud, private cloud, hybrid cloud, cloud service provider, workloads, and service level agreements. In that, customers demand the service without knowing about the backside process of clouds and pay as per that demand. The public cloud consists of the assets that are available for all at free of cost or as pay per demand model. A private cloud consists of the assets that are not available for all the customers, only available to that user who owns the cloud. A hybrid cloud is formed by the partnership made between the public and private clouds. Cloud service providers like Amazon EC2, Microsoft Azure, and Google provide assets as per the demand of customers. A federated cloud environment contains a workload generated by different applications and facilities engaged in cloud infrastructures. In short, cloud workload is nothing but a combination of tasks given by the various customers of the cloud. Service Level Agreement signed between the cloud service providers and customers to maintain the Quality of Service (QoS) [20].

Federated clouds follow different coupling levels for asset monitoring, asset collaboration, remote governing, etc. Coupling levels in federated cloud environments are majorly categorized into three types such as loose coupling, partial coupling, and tight coupling.

Loose Coupling - in this level of coupling only basic operations that are less complex are carried out. More complex operations like asset migrations are not carried out at this level. Basic functions on virtual machines, assets monitoring, and remote governing are only done at this level. This level provides security at a single cloud at the organization level only.

Partial Coupling - In this level of coupling more than single cloud service providers share their assets according to their terms and conditions. At this level, basic operations like virtual machine maintenance and asset controlling are done. This level provides security according to the agreement framework.

Tight Coupling - in this level of coupling complex operations like scheduling of certain assets, virtual machine migration, and remote monitoring are done. This level provides security at the customer region level.

\section{FEDERATED CLOUD ARCHITECTURE}

Federation of clouds collaborates with different cloud service providers and facilities provided by them as a single unit to fulfill customer's demands. Federated cloud computing has two basic dimensions such as horizontal federation and vertical federation. In that, horizontal federation is seen at only on one single level that is nothing but in the form of cloud stack or application stack, whereas another one vertical federation seen at multiple levels. These two horizontal and vertical scaling of the cloud federation environment improves the application performance and restoration of the cloud resources. For example addition of extra disk, virtual CPU, and RAM to efficiently handle increased burden on applications in the vertical federation.
The horizontal federation needs more computing devices for the fulfillment of increased customer demands.

Federated cloud architecture is shown in the following Figure 1 that contains three main basic components. Such as cloud exchange, cloud coordinator, and cloud broker.

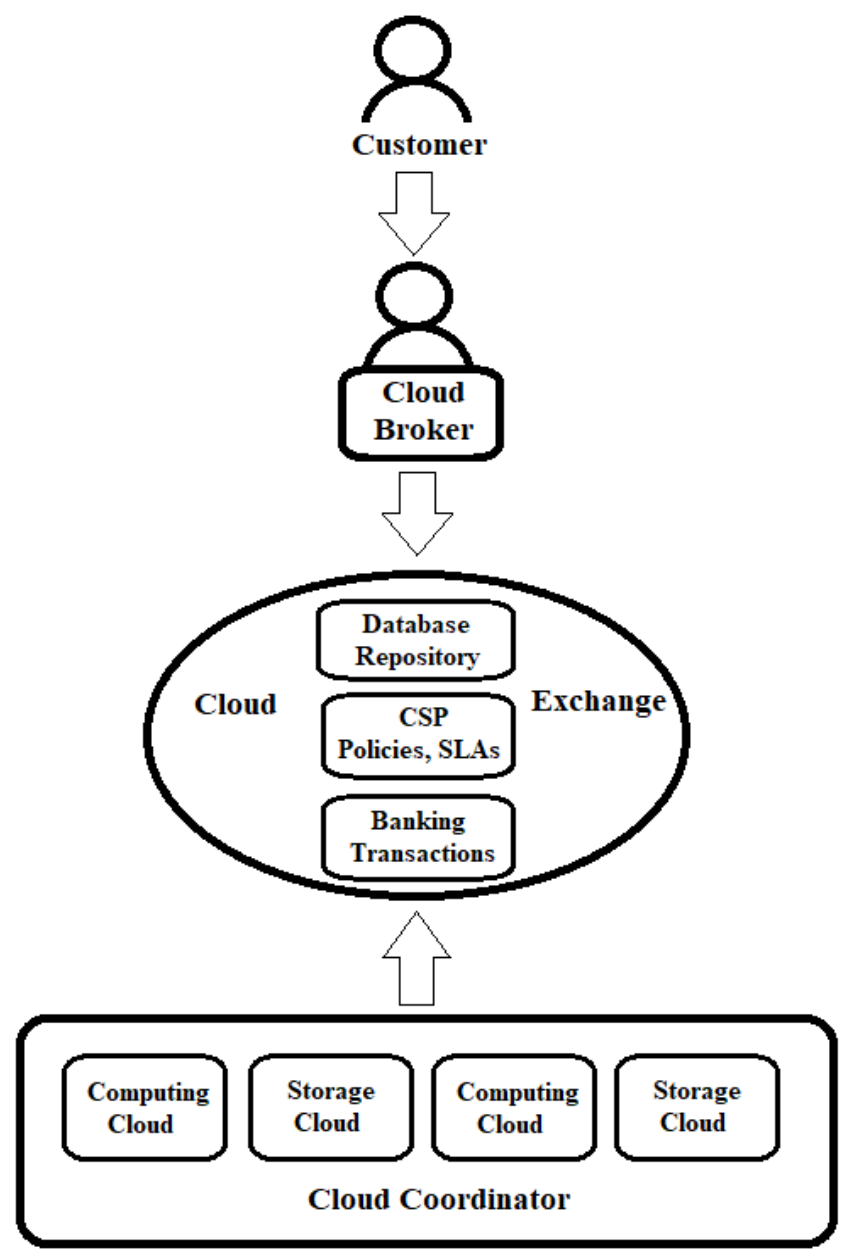

Fig.1. Federated Cloud Architecture

Cloud Exchange - It acts as an intermediate between the cloud broker and cloud coordinator. As an intermediary between broker and coordinator, cloud exchange maps the demands from the cloud broker to the available facilities provided by the cloud coordinator. The cloud exchange keeps the record of currently available cloud service providers, request patterns, and the current price of facilities maintained at the database repository. The cloud broker always communicates with cloud exchange to get information regarding the current SLA strategies and the number of available resources from various cloud service providers. The cloud exchange enables, maintains, and handles all the banking transactions between the cloud service providers and their customers to maintain transparency and trust.

Cloud Coordinator - All the information stored at the cloud exchange database repository is updated by the cloud coordinator on a timely basis.

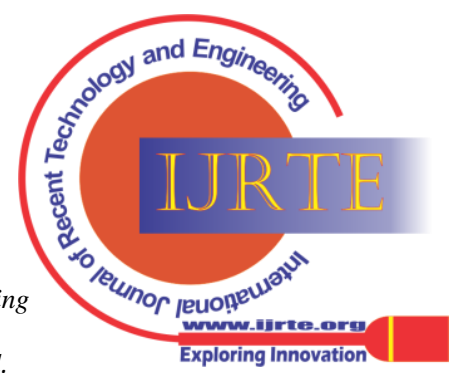


The cloud coordinator allocates the resources of the cloud to the customer based on the Quality of Service (QoS) they request and according to their budget. The cloud coordinator develops the pricing and marketing models based on the Service Level Agreement (SLA). It also manages the cloud enterprises and their membership.

Cloud Broker - On behalf of the customer cloud broker, is the entity that interacts with the cloud exchange to obtain information regarding pricing and marketing models, SLA rules, available resources, and cloud service providers. After reviewing these entire things carefully the cloud broker finalizes the best suitable contract for their customer.
Federated cloud is further categorized into four different types, such as cloud bursting architecture, cloud broker architecture, aggregated cloud architecture, and cloud multitier architecture.

\section{A. Cloud Bursting Architecture}

This type of federated cloud architecture was formed when resource exhaustion happened in the internal network. In this architecture, workloads burst from private cloud to public cloud. This architecture follows a loose coupling level. Hence, only basic operations that are less complex are carried out. More complex operations like asset migrations are not carried out in this type of cloud-bursting architecture. Figure 2 shows the cloud bursting architecture.

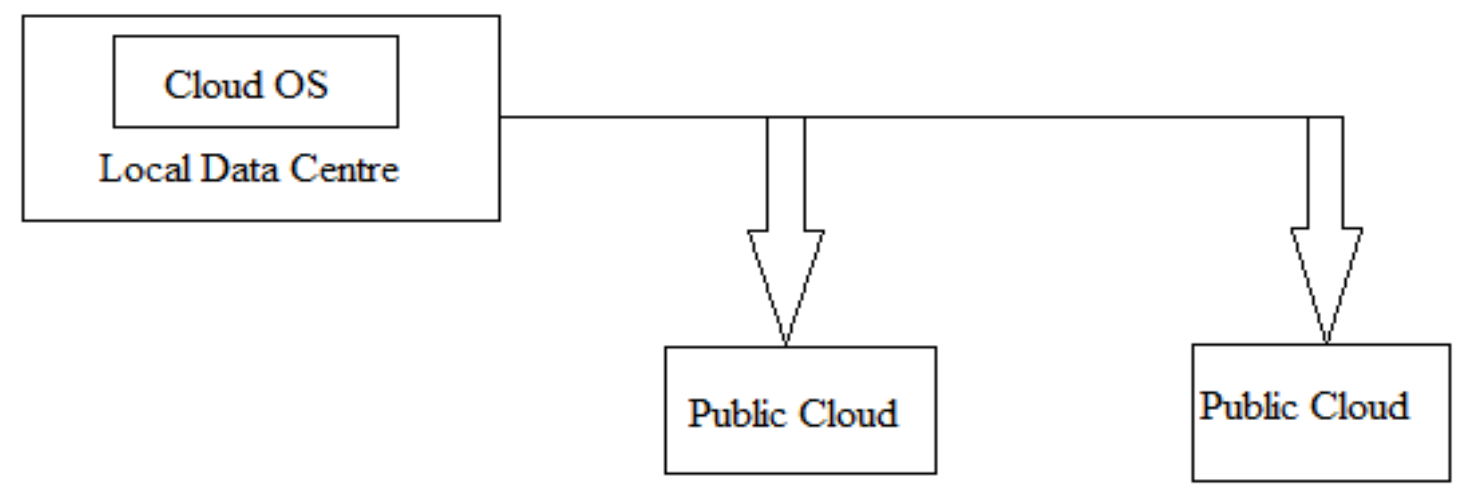

Fig.2. Cloud Bursting Algorithm [20]

\section{B. Cloud Broker Architecture}

This type of federated cloud architecture was formed to optimize the cost and to improve request execution time. In this type of architecture, a cloud broker takes all the responsibilities to choose the best cloud service provider who can fulfill the customer's demand at a good price rate. In this architecture, the cloud broker acts as an intermediate between the customer and the cloud service provider. This type of cloud broker hides all the management difficulties and reduces the difficulties of the customer who can't make direct communication with the cloud service providers because of terms and conditions. This architecture also follows a loose coupling level. Figure 3 shows the cloud broker architecture.

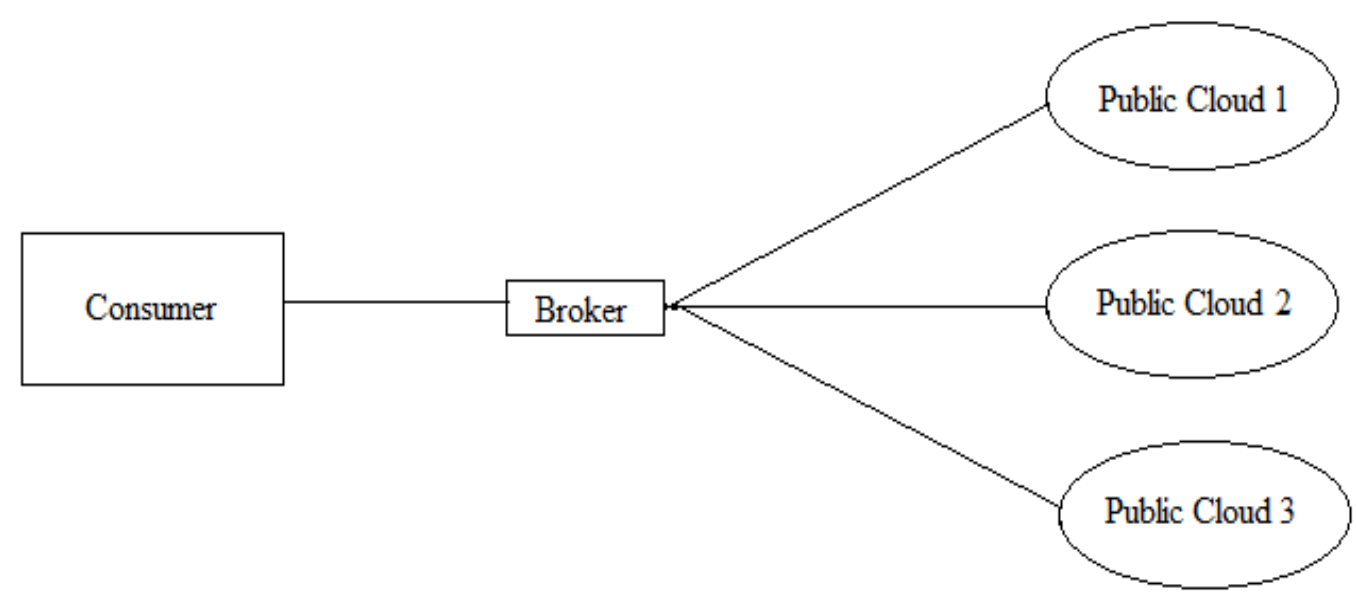

Fig.3. Cloud Broker Architecture [20]

\section{Aggregated Cloud Architecture}

This type of architecture was formed to meet the customer requirements through resource sharing. In this type of architecture hybridization of public and private clouds takes place to fulfill the customer demands. Hence, different cloud service providers aggregate their assets based on agreements and frameworks to fulfill the customer demand individually. Here also cloud brokers choose the best cloud service provider for particular customer demand. This architecture follows a partial coupling level. Hence, basic operations like asset maintenance and controlling are carried out and provide security according to the agreement framework. Figure 4 shows the aggregated cloud architecture.

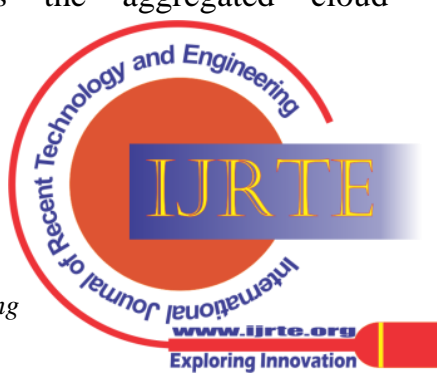




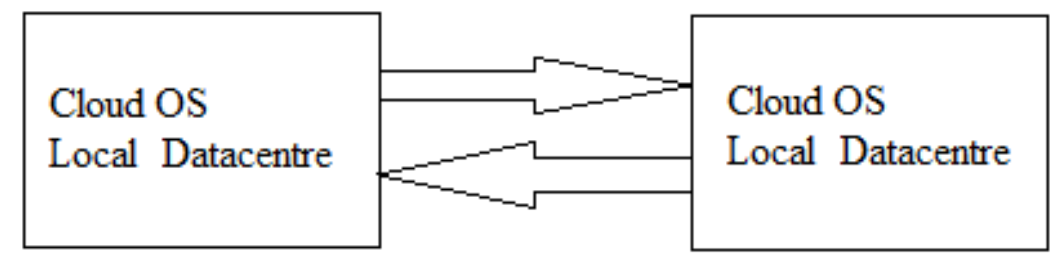

Fig.4. Aggregated Cloud Architecture [20]

\section{Cloud Multi-tier Architecture}

In this type of cloud architecture large public and private cloud data centers combined with multiple other data centers due to which infrastructure assets can be accessed from any of the data centers. This architecture is the most scalable form among all other types of federated cloud architectures. This architecture follows a tight coupling level. Hence, complex operations like scheduling, migration, and remote monitoring of assets are carried out and provide security at the customer region level. In this type of architecture, various resources are scattered at different geographical locations, the price of a separate coordinator system is more as compared with other types of federated cloud architecture. Figure 5 shows the cloud multi-tier architecture.

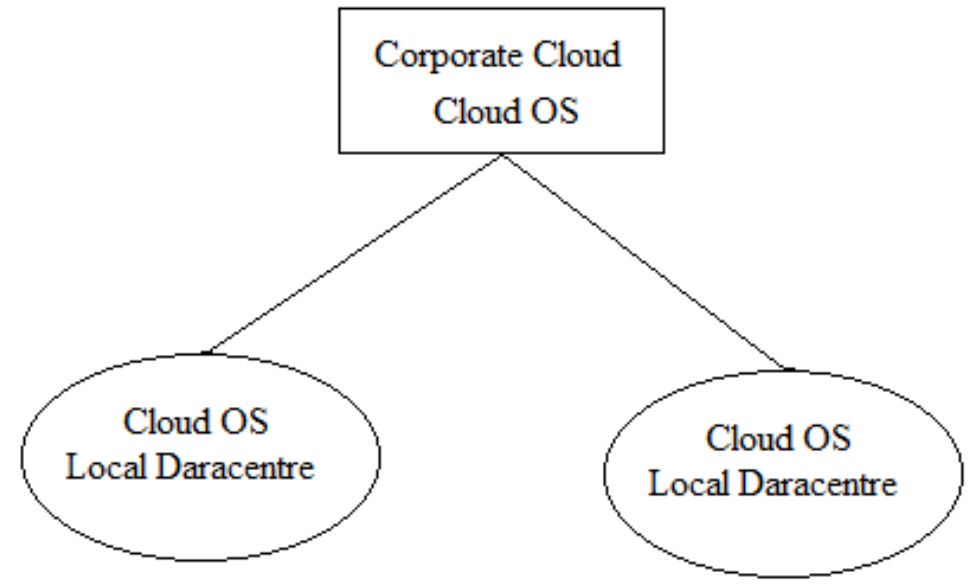

Fig.5. Cloud Multi-tier Architecture [20]

\section{ADVANTAGES OF FEDERATED CLOUD COMPUTING}

A federated cloud environment has a lot of benefits over simple cloud computing and single deployed cloud in terms of multi-cloud deployment, guaranteed availability, scalability, fault tolerance, avoidance of vendor lock-in, and performance.

Multi-cloud deployment - In a federated cloud environment, several cloud resources are aggregated together from different cloud service providers to fulfill the demands of the customer that further optimizes the cost of facilities.

Availability - In a federated cloud environment any natural calamity, any unexpected disasters, and any technical trouble can't affect its cloud facilities due to federation with multiple cloud service providers situated at different geographical locations all over the world.

Scalability - In a federated cloud environment, if demands from customers increase at any time, any cloud service providers can share their computing resources and facilities through a cloud service broker which gives large scalability to this cloud system.

Fault tolerance - In a federated cloud environment, stored data of customers is also duplicated somewhere in the cloud network. Hence, any catastrophe like a server down or any natural calamity can't trigger data loss or no data in this cloud system.
Avoiding vendor lock-in - In a federated cloud environment, customers can readily transfer their workload among any cloud according to their requirements hence also avoid vendor lock-in swiftly. If any cloud service provider changes the terms and conditions or any rule and the customer is not happy with it, then the customer can easily shift to another cloud service provider to avoid the adverse effect of that changed condition.

Performance - In a federated cloud environment not only optimize the cost value of facilities but also minimizes the execution time or response time needed to fulfill the demand of customers, which is directly reflected in the form of increased system throughput, efficient use of resources, and improved performance of the cloud system.

\section{CHALLENGES IN FEDERATED CLOUD}

A federated cloud environment consists of complexity, heterogeneity due to different cloud types, numerous cloud service providers, and their dynamic cloud systems. All that versatility causes various challenging issues in a federated cloud environment such as portability, interoperability, deployment strategy, security, Quality of Service, pricing model, and SLA.

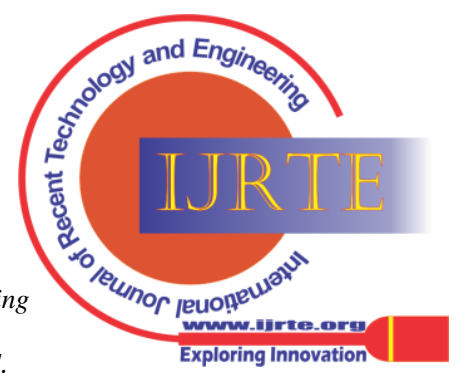


Portability - Customer demands rise considerably and to fulfill those demands without any delay is a very important aspect in federated cloud. Hence, there is a need to combine two or more numbers of public and private clouds that are nothing but a federation of clouds. In such a scenario sometimes data also needed to be transferred from one cloud data center to another cloud data center securely. This indicates the importance of the portability issue in a cloud federation.

Interoperability - When heterogeneous clouds form federation then interoperability acts as the main concept. In a cloud federation, every cloud consists of its interfaces for facilities and its solutions for problems. In simple cloud computing customers are locked into a single cloud infrastructure, application, or platform and avoids data portability and software portability. Hence, in cloud federation infrastructure issues need to be considered while generating a platform for interoperability among different cloud service providers. There is a need to construct standard interfaces that can interact with various distributed sites.

Deployment Strategy - As general cloud service providers give various computing resources according to the demands of the customers. The cloud broker selects the best computing resources that can fulfill the demand of customers proficiently. But this can't happen all the time due to uncertainty in customer demands. Sometimes customer demands less often. Hence, during this off-peak demand time maintaining the computing resources properly is various tiresome works for cloud service providers.

Security - As usual in a federated cloud environment every cloud uses different security, identity management, audit process, and authentication measures. Hence, security issues needed to be solved while combining two or more different types of clouds in a federation, to provide better authentication among all the heterogeneous clouds.

Quality of Service (QoS) - QoS generally depends on the SLA made between the cloud service provider and customer. It plays an important part to fulfill the customer's demand. When computing resources are drained because of any reason like natural calamities or the high number of customer demands there is no need to break SLA by compromising the QoS.

Pricing Model - Different cloud service providers follow their pricing model in a federated cloud environment based on what kind of facilities they delivered. This will cause a lot of confusion from the customer's point of perspective. To avoid all this there should be one uniform pricing model that needs to be followed in a federated cloud environment.

Service Level Agreement (SLA) - In a federated cloud environment, every cloud service provider has its SLA scheme. Due to which every action performed in the federated cloud was carried out very carefully. Such as several clouds sharing their computing resources, managing cloud facilities through API without breaking SLAs. To avoid all these there is a need to develop a global level SLA, which includes all SLAs of cloud service providers in the federation and acts as broad SLAs between the customer and all federated cloud service e providers. demands from customers are too many and sometimes

\section{FUTURE RESEARCH DIRECTIONS}

There are a few research areas in cloud federation that can be improved in the future to make a federation environment more beneficial. The goal of this survey paper is to focus on various factors like viability and the assistance is helpful to improve the quality of service and involvement by using federated cloud computing. To improve the utilization of facilities, tackle dynamic processing loads and performance enhancements are the main goals of this work. So to achieve all these goals some areas need specific directions. In the resource scheduling process in a federated environment cloud brokers can predict workload before handling any particular customer demand and can select the best cloud service provider. This kind of arrangement improves the response, execution time, and any delay. Further cloud brokers can select the computing resources from particular cloud service providers based on the location which is beneficial to the customer, to minimize the delay. In a federated environment, every cloud service provider gives SLAs based on their computing assets, to improve the performance by optimizing the assets. SLAs can be designed according to any application and as per the requirements of the customers due to which it would be beneficial to both customers and cloud service providers.

\section{CONCLUSION AND FUTURE SCOPE}

Federated cloud computing terminology has a big perspective and tremendous impact on computing assets and applications. It is the next way to provide computing resources to utility-based services. In a federated cloud environment, the cloud broker act is an intermediary between customers and cloud service providers to select the best service provider. However, this cloud broker does not follow an application-based methodology but can maintain the quality of service in a federated cloud environment. The resource scheduling mechanism arranges workloads to different assets selected by the cloud broker. Cloud bursting offers optimization of price from the cloud service provider's side by exhausting the internal cloud assets. This work gives in a detailed study of federated cloud computing, cloud federation environment, various types of federated cloud computing architectures, benefits of federated cloud computing, various challenging issues of a federated cloud environment, and finally some future research directions which will make federated cloud environment more beneficial in coming days. On the other hand, the advancement of federated cloud computing and the technologies associated with it are tremendously dynamic. Hence it is very difficult to make any long-term projections. Hopefully, this will contribute to future research works for industrial and also for academic purposes in the cloud federation area of research.

\section{REFERENCES}

1. M. Armbrust, A. Fox, R. Griffith, A. D. Joseph, R. Katz, A. Konwinski, G. Lee, D. Patterson, A. Rabkin,I. Stoica, M. Zaharia, "Above the clouds: A Berkeley View Of Cloud Computing", University of California at Berkeley, Tech. Rep., February 2009, [Online].

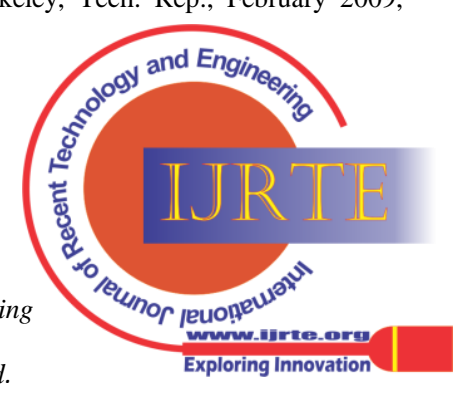


2. B Rochwerger et al "The Reservoir Model and Architecture for Open Federated Cloud Computing", in proceedings of the IBM Journal of Research and Development, Volume No. 53, Issue No. 4, July 2009, pp. 4:1-4:11.

3. R. Van den Bossche, K. Vanmechelen, J. Broeckhove "Cost-Optimal Scheduling in Hybrid IAAS Clouds for Deadline Constrained Workloads", in proceedings of the 3rd IEEE International Conference on Cloud Computing (CLOUD), Miami, USA, 5-10 July 2010, pp. 228-235.

4. Tobias Kurze, Markus Klems, David Bermbach, Alexander Lenk, Stefan Tai and Marcel Kunze "Cloud Federation", proceedings of the 2nd International Conference on Cloud Computing, GRIDs and Virtualization, January 2011, pp. 32-38.

5. David Breitgand, Alessandro Maraschini, Johan Tordsson "PolicyDriven Service Placement Optimization in Federated Clouds", IBM Research, Computer Science, Volume No. 12, March 2011, pp. 1102-14.

6. RB Bohn, J Messina, F Liu, J Tong, J Mao "NIST Cloud Computing Reference Architecture" in proceedings of the IEEE World Congress on Services, Volume No. 1, Washington, DC USA, 4-9 July 2011, pp 594-596.

7. Christian Vecchiola, Rodrigo N. Calheiros, Dileban Karunamoorthy, Rajkumar Buyy "Deadline-Driven Provisioning of Resources for Scientific Applications in Hybrid Clouds with Aneka", Future Generation Computer Systems, Volume No. 28, Issue No. 1, January 2012, pp 58-65.

8. Johan Tordsson, Rubén S.Montero, Rafael Moreno-Vozmediano, Ignacio M.Llorente "Cloud Brokering Mechanisms for Optimized Placement of Virtual Machines Across Multiple Providers", Future Generation Computer Systems, Volume No. 28, Issue No. 2, February 2012, pp. 358-367.

9. S. Chaisiri, B. Lee, D. Niyato "Optimization of Resource Provisioning Cost in Cloud Computing", in proceedings of the IEEE Transactions on Services Computing, Volume No. 5, Issue No. 2, April-June 2012, pp. 164-177.

10. Peter Wright, Yih Leong Sun, Terence Harmer, Anthony Keenan, Alan Stewart, Ronald Perrott "Constraints-Based Resource Discovery Model for Multi-Provider Cloud Environments", Journal of Cloud Computing: Advances, Systems, and Applications, Volume No.1, Article No. 6, 21 June 2012.

11. Bahman Javadi, Jemal Abawajy, Rajkumar Buyya "Failure-Aware Resource Provisioning for Hybrid Cloud Infrastructure", Journal of Parallel and Distributed Computing, Volume No. 72, Issue No. 10 , October 2012, pp. 1318-1331.

12. Rodrigo N. Calheiros, Adel Nadjaran Toosi, Christian Vecchiola, Rajkumar Buyya "A Coordinator for Scaling Elastic Applications Across Multiple Clouds", Future Generation Computer Systems, Volume No. 28, Issue No. 8, October 2012, pp. 1350-1362.

13. Ghazizadeh Eghbal, Jamalul-lail Ab Manan, Zamani Mazdak, Pashang Abolghasem "A Survey on Security Issues of Federated Identity in the Cloud Computing", in proceedings of the 4th IEEE International Conference on Cloud Computing Technology and Science (CloudCom-2012), December 2012, pp. 532-565.

14. Mark Badger, Tim Grance, Robert Patt-Corner, Jeffrey Voas "Cloud Computing Synopsis and Recommendations", NIST Special Publication (NIST SP), December 2012, pp. 800-146.

15. Jose Luis Lucas-Simarro, Rafael Moreno-Vozmediano, Ruben S Montero, Ignacio M. Llorente "Scheduling strategies for optimal service deployment across multiple clouds", Future Generation Computer Systems, Volume No.29, Issue No. 6, August 2013, pp. 1431-1441.

16. Osama Shareef, Ahmad Kayed "A Survey on Federated Clouds Environment", International Journal of Advanced Research in Computer Science and Software Engineering (IJARCSSE), Volume No. 5, Issue No. 2, February 2015, pp. 83-92.

17. Shishira SR, Kandasamy A, Chandrasekaran K "Workload Scheduling in Cloud: A Comprehensive Survey and Future Research Directions", in proceeding of the 7th IEEE International Conference on Cloud Computing, Data Science \& Engineering (Confluence2017), Noida, India, January 2017, pp. 269-275.

18. Moustafa Najim, Venkatesh Tamarapalli "A Cost-Aware Algorithm for Placement of Enterprise Applications in Federated Cloud Data Center", in Proceedings of the 20th International Conference on Distributed Computing and Networking (ICDCN 2019), Bangalore, India, 4-7 January 2019, ACM, pp. 510.

19. V. Keerthi, T. Anuradha "A Study on Federation Clouds and its Issues", International Journal of Computer Sciences and Engineering (IJCSE), Volume No. 8, Issue No. 3, March 2020, pp. 109-113.

20. S. R. Shishira and A. Kandasamy "A Comprehensive Survey on Federated Cloud Computing and its Future Research Directions",
Evolutionary Computing and Mobile Sustainable Networks, in proceedings of the ICECMSN 2020, V. Suma et al.(eds.) Lecture Notes on Data Engineering and Communications Technologies 53, Springer Nature Singapore Pte Ltd.2021, pp. 79-88.

\section{AUTHORS PROFILE}

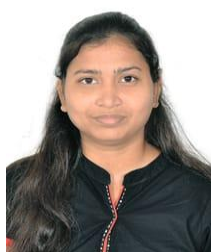

Sapana Sanjay Bhuskute, is currently working as an Assistant Professor at the Departments of BSc IT \& CS at ICLES Motilal Jhunjhunwala College of Arts, Science and Commerce, Vashi. She is pursuing her ME from Terna Engineering College, Nerul, Navi Mumbai. Degree BE in Information Technology completed from Saraswati College of Engineering, Kharghar in 2016, with the First Class. She has four years of academic experience at the University of Mumbai. Her research activities involve Cloud Computing, Data Security, and Data Mining. She has published one research paper on IEEE Digital Library through the 5th IEEE International Conference on Intelligent Computing and Control Systems (ICICCS -2021)

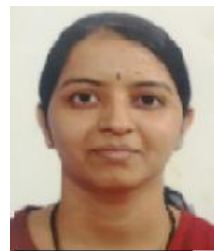

Sujata R Kadu, is currently working as an Assistan Professor at the University of Mumbai, Terna Engineering College. She received her B. Tech degree from Dr. Babasaheb Ambedkar Technological University in 2003 and ME degree from the University of Mumbai in 2009 from Electronics and Telecommunication. She is currently working towards a Ph.D. degree from the University of Mumbai. Her research areas include Signal Processing, Image Segmentation and Classification, Object-Based Image Analysis, and Multiresolution Segmentation.

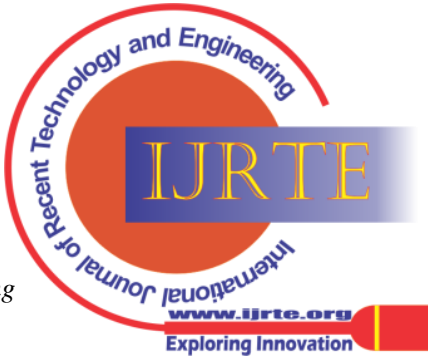

